\section{REPURPOSING CD26 (DPP4) INHIBITORS TO ENHANCE IMMUNOTHERAPY RESPONSE IN PANCREATIC DUCTAL ADENOCARCINOMA}

${ }^{1}$ Maggie Phillips*, 'Michael Ware, ${ }^{1}$ Cameron Herting, ${ }^{2}$ Thomas Mace, 'Shishir Maithel, ${ }^{1} J u a n$ Sarmiento, ${ }^{1}$ Bassel El-Rayes, ${ }^{1}$ Chrystal Paulos, ${ }^{1}$ Megan Wyatt, ${ }^{1}$ Gregory Lesinski. ${ }^{1}$ Emory University, Atlanta, GA, USA; ${ }^{2}$ OSUMC, Columbus, OH, USA

Background Pancreatic ductal adenocarcinoma (PDAC) is refractory to immunotherapy due in part to cellular cross-talk with cancer associated fibroblasts (CAFs). These interactions shape the microenvironment in a manner that is profoundly immunosuppressive. Our group is identifying novel targets in the PDAC stroma that can be manipulated to enhance immunotherapy efficacy. We hypothesize dysregulation of the serine protease, CD26/DPP4 in PDAC contributes to the limited efficacy of immunotherapy. Further, we posit targeting CD26 enzymatic activity using inhibitors that are FDA-approved for adult patients with Type 2 Diabetes Mellitus can enhance the efficacy of immunotherapy in PDAC.

Methods Primary CAFs isolated from patient PDAC resection specimens under an IRB-approved protocol, were subject to NanoString analysis. ${ }^{1}$ CD26 protein expression was measured in primary and immortalized CAFs and PDAC cells by immunoblot, flow cytometry and immunofluorescence, while ELISA detected soluble CD26. For in vivo efficacy, luciferase-expressing KPC-tumor cells were implanted orthotopically in the pancreas of immune-competent C57BL/6 mice. Bioluminescence imaging (BLI) confirmed established tumors and mice were randomized to sitagliptin $(75 \mathrm{mg} / \mathrm{kg}$ in drinking water, CD26/ DPP4 inhibitor), anti-PD-L1 Ab (200 ug 2x/week), or both combined for 3 weeks. Controls received vehicle or isotype control $\mathrm{Ab}$. BLI utilized to track tumor progression and tissues harvested for analysis at study endpoint (day 18 of treatment). Results NanoString analysis identified CD26/DPP4 as significantly upregulated in RNA transcripts from primary CAFs vs. fibroblasts from normal pancreas (figure 1). We confirmed abundant CD26 expression on patient-derived CAFs and immortalized CAF cell lines, however, lower CD26 expression was observed on human PDAC cell lines (HPAC, PANC-1) by immunoblot, flow cytometry and immunofluorescence (figure 5).

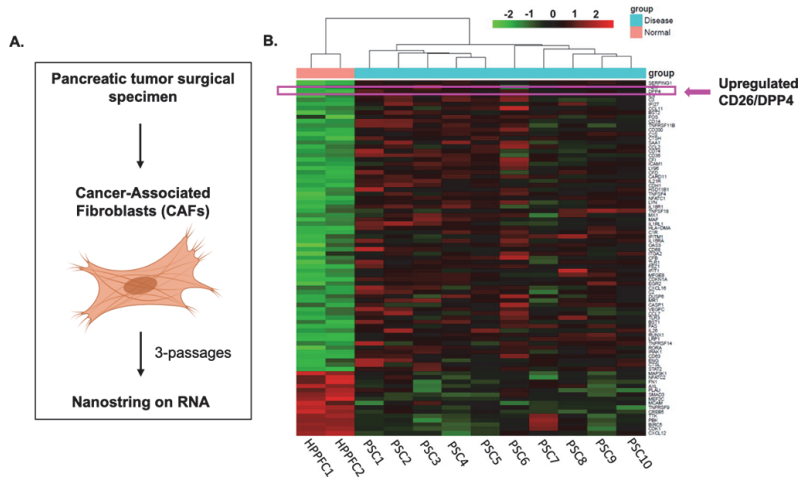

Abstract 904 Figure 1 (A) Schema for analysis of transcript from $\mathrm{n}=10$ primary CAFs (PSC) from PDAC patients vs. normal human pancreatic fibroblasts (HPPFC) via NanoString nCounter PanCancer Immune Profiling Panel. (B) Heat map of gene expression with upregulate DPP4 or CD26 transcript detected. Adapted from Mace et al., 2016.

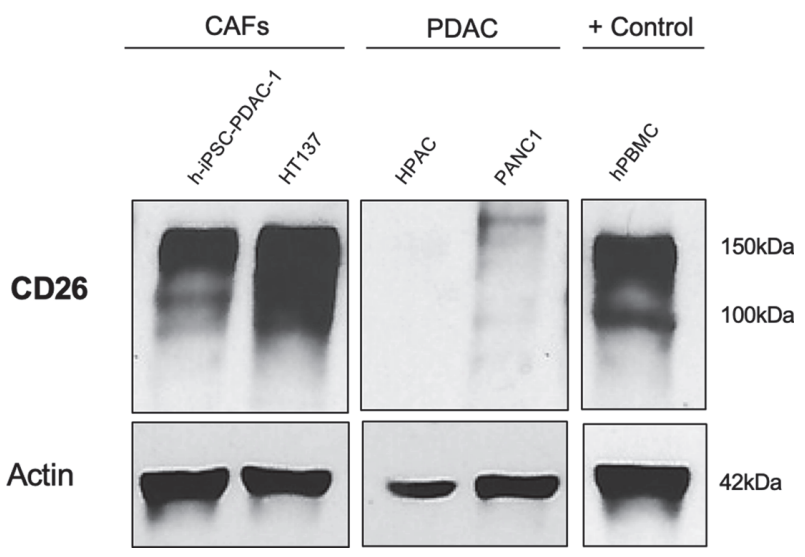

Abstract 904 Figure 2 Validation of CD26 protein expression in human PDAC-derived CAF and PDAC cell lines by immunoblot analysis

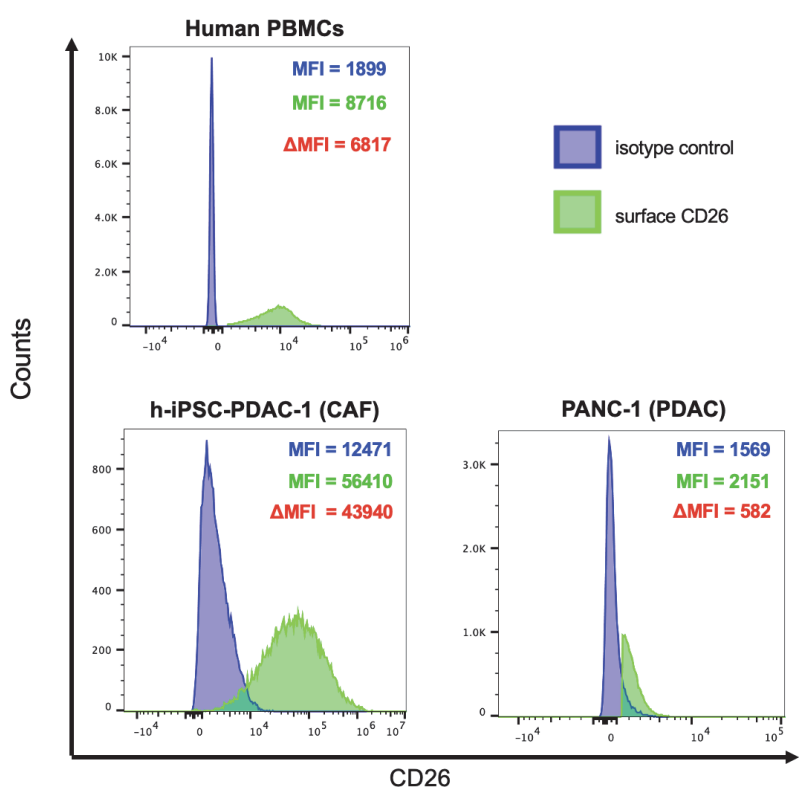

Abstract 904 Figure 3 Analysis of surface human CD26 expression in PBMCs, PDAC-derived CAFs (h-iPSC-PDAC-1), and PDAC cells (PANC-1) by flow cytometry. Histograms representing human surface CD26 expression
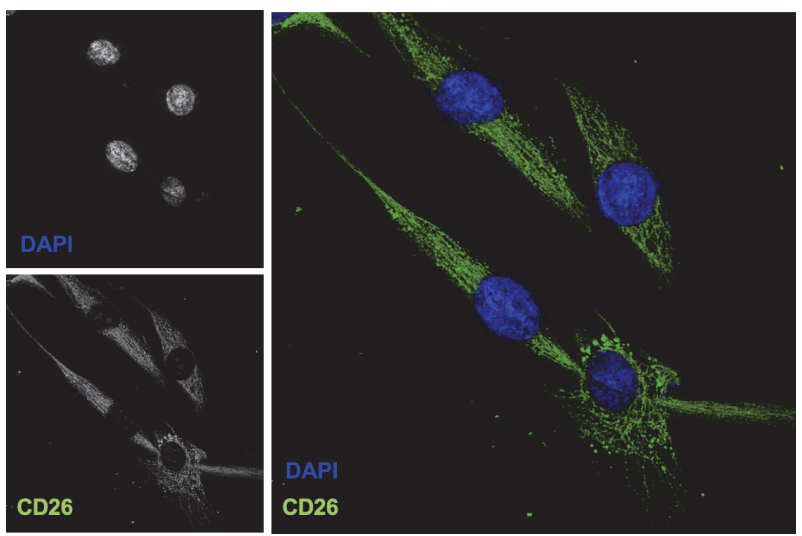

Abstract 904 Figure 4 Immunofluorescence analysis of CD26/DPP4 cellular localization in a human PDAC-derived CAF cell line 


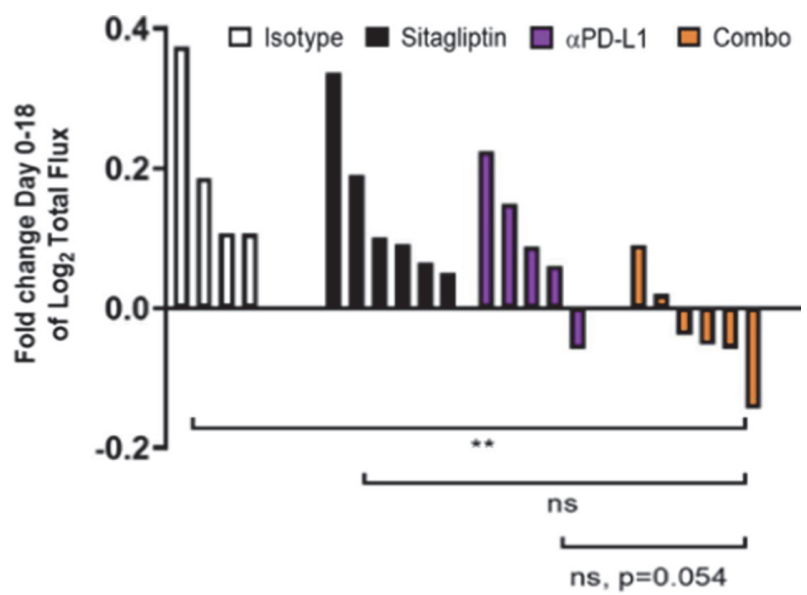

Abstract 904 Figure 5 Combined Sitagliptin and PD-L1 blockade in a murine orthotopic model of PDAC. Fold change in tumor volume, determined by BLI, comparing baseline (Day 0 of treatment) to Day 18 of treatment. Each bar represents fold change in BLI determined tumor volume for each animal

Conclusions Our results are the first to describe CD26 expression on PDAC-derived CAFs and indicate that sitagliptin augments anti-tumor activity of anti-PD-L1 in PDAC tumorbearing mice. Our ongoing work will provide insight into specific immune cell populations responsible for efficacy of immunotherapy in murine models of PDAC, and the role of CD26 in various cellular compartments within the PDAC microenvironment.

\section{REFERENCES}

1. Mace TA, Shakya R, Pitarresi JR, Swanson B, McQuinn CW, Loftus S, Nordquist E, Cruz-Monserrate Z, Yu L, Young G, Zhong X, Zimmers TA, Ostrowski MC, Ludwig T, Bloomston M, Bekaii-Saab T, Lesinski GB. IL-6 and PD-L1 antibody blockade combination therapy reduces tumour progression in murine models of pancreatic cancer. Gut 2018;67(2):320-32.

http://dx.doi.org/10.1136/jitc-2021-SITC2021.904 